\title{
Gelastic Seizures and Hypothalamic Hamartoma
}

\author{
Otto Jesús Hernández Fustes ${ }^{1,2}$ \\ ${ }^{1}$ Serviço de Neurologia, Universidade Federal do Paraná, Hospital \\ de Clínicas, Curitiba, PR, Brasil \\ 2 InNeuro, Hospital das Nações, Curitiba, PR, Brasil \\ ${ }^{3}$ Departamento de Medicina, Universidade Positivo, Curitiba, \\ PR, Brasil
}

J Neurosci Rural Pract 2020;11:675-675

In 1877, Trousseau first described a laughter crisis in a young man. In 1957, Daly and Mulder proposed the term gelastic seizures (GS), from the Greek word gelos meaning "laughter," emphasizing this condition's the main characteristic., ${ }^{1,2}$

The accepted diagnostic criteria for GS include recurrent stereotyped fits of laughter, the absence of precipitating external factors, laughter inappropriate to context, laughter concomitant with other epileptic clinical manifestations, and ictal or interictal epileptiform electroencephalographic (EEG) changes. ${ }^{3}$

GS are frequently associated with hypothalamic hamartomas (HHs), rare non-neoplastic congenital malformations composed of ectopic neurons and glial cells. HH has an estimated prevalence of 1 in 50,000 to 100,000, more frequently in males. $\mathrm{HH}$ occurs concurrently with precocious puberty in $63 \%$ of patients, epileptic seizures in $61 \%$, and both in $25 \% .4,5$

We present a case of GS associated with an $\mathrm{HH}$ in a 19-year-old male with retarded psychomotor development. At the age of 4 years, he presented with short-duration disconnection crises (focal impaired-awareness seizures) followed by generalized tonic-clonic seizures that were refractory to multiple antiepileptic drugs (AEDs). The seizures morphed, at 10 years, into focal seizures of unmotivated, uncontrollable, and explosive laughter lasting for seconds without precipitating factors and occurring several times a day. Examination revealed slow, monotonous, and monosyllabic language without other peculiarities. EEG showed acute paroxysmal left temporoparietal and right temporal interictal epileptiform waves and right temporal ictal epileptiform activity. A computed tomography (CT) scan of the brain was normal. Magnetic resonance imaging (MRI) of the brain revealed a right hypothalamic mass with spectroscopy compatible with an $\mathrm{HH}$. There was partial seizure
Address for correspondence Otto Jesús Hernandez Fustes, MD, MSC, Hospital de Clínicas da Universidade Federal do Paraná, Rua General Carneiro 181, 80060-900, Curitiba/PR, Brasil (e-mail: otto.fustes@hc.ufpr.br).

control, without the occurrence of tonic-clonic seizures, and a $70 \%$ decrease in the frequency of focal and GS with the use of carbamazepine $1200 \mathrm{mg} /$ day.

$\mathrm{HH}$ is associated with endocrine and neurological manifestations, commonly occurring in childhood with generalized early-onset dysperceptive and tonic seizures that are resistant to AEDs. ${ }^{5}$ The onset of seizures in adolescence, as in our case, is infrequent; however, the refractory behavior of GC was similar to previous reports. The EEG supported the diagnosis of epilepsy and was hemispherically related to the lesion on brain MRI. We deduced that an HH had caused all crises in the patient, including the intellectual deficit and developmental delay of school skills.

Our patient emphasizes the need for imaging, preferably brain MRIs, in patients with epileptic seizures due to the 14 -year delay in identifying the etiology of epilepsy.

\section{Conflict of Interest}

None declared.

\section{References}

1 Trousseau A. De L'Epilepsie. Clinique Medicale de L'Hotel-Dieu de Paris 1877;89-155

2 Daly DD, Mulder DW. Gelastic epilepsy. Neurology 1957; 7(3):189-192

3 Gascon GG, Lombroso CT. Epileptic (gelastic) laughter. Epilepsia 1971;12(1):63-76

4 Conde Blanco E, Anciones Martín C, Manzanares I, et al. Hypothalamic hamartomas in adulthood: clinical spectrum and treatment outcome-a unicenter experience. Brain Behav 2019;9(11):e0141210.1002/brb3.1412

5 Nguyen D, Singh S, Zaatreh M, et al. Hypothalamic hamartomas: seven cases and review of the literature. Epilepsy Behav 2003;4(3):246-258 